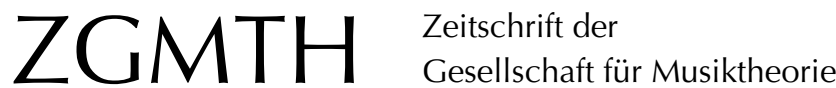

Diergarten, Felix (2003/05): Riemann-Rezeption und Reformpädagogik. Der Musiktheoretiker Johannes Schreyer. ZGMTH 1-2/2/1, 163-170.

https://doi.org/10.31751/466

(C) 2003/05 Felix Diergarten

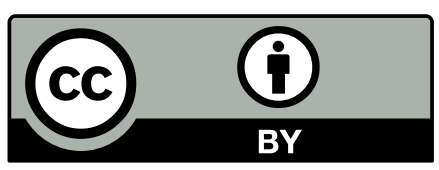

Dieser Text erscheint im Open Access und ist lizenziert unter einer Creative Commons Namensnennung 4.0 International Lizenz.

This is an open access article licensed under a Creative Commons Attribution 4.0 International License.

veröffentlicht / first published: 01/01/2005

zuletzt geändert / last updated: 15/09/2009 


\title{
Riemann-Rezeption und Reformpädagogik
}

\section{Der Musiktheoretiker Johannes Schreyer}

\author{
Felix Diergarten
}

Im Sommer des Jahres 1903 erschien in Dresden die Harmonielehre von Johannes Schreyer. Sie gehörte zu den meistgelesenen Harmonielehren ihrer Zeit. Ein zeitgenössischer Kritiker bezeichnete sie sogar als »das Beste, was wir auf dem Gebiet der Harmonielehre besitzen«. Schreyers Harmonielehre stellt einen der ersten Versuche dar, die Riemannsche Harmonie- und Phrasierungslehre zu einem praktischen Lehrgang umzuformen. Sie ist in ihrem Aufbau und ihrer Didaktik durch die `Reformpädagogikı geprägt und wurde als eine der in theoretischer und didaktischer Hinsicht fortschrittlichsten Harmonielehren ihrer Zeit begrüßt. Unter Analyse versteht Schreyer in erster Linie das Anfertigen reduktionistischer Harmonieskizzen. Die zahlreichen Musikbeispiele aus der Literatur von Palestrina bis Reger werden mit Riemannschem Klangschlüssel und Funktionszeichen beziffert. Der Begriff von `Funktion`, den Schreyer dabei entwickelt, zeigt sich in erstaunlicher Nähe zum Begriff der 'Stufe`, den Heinrich Schenker wenige Jahre später prägen sollte. Das Werk Schreyers ist jedoch nicht nur ein eindrucksvolles Zeugnis früher Riemann-Rezeption; es zeigt sich an der Harmonielehre Schreyers darüber hinaus, zu welchem Zeitpunkt und in welcher Form Lebensphilosophie und Kulturkritik immensen Einfluß auf die deutsche Musiktheorie und den gesamten deutschen Musikbetrieb gewinnen konnten.

\section{Riemann-Rezeption}

Der eigentliche Reformator für unsere Zeit bleibt Riemann. Auch Johannes Schreyer ist ohne ihn nicht denkbar. Trotzdem beansprucht seine Harmonielehre einen Platz für sich wegen ihres Zieles und wegen der Wege zu diesem Ziele. (Georg Göhler)

Zu Beginn des 20. Jahrhunderts übte auf dem Gebiet der Harmonielehre in Deutschland wohl niemand einen vergleichbaren Einfluß aus wie Hugo Riemann (vgl. Holtmeier 2004). Wer auch immer sich nach Riemann über Fragen der Harmonielehre äußerte, mußte in irgendeiner Weise zu ihm Stellung beziehen, und das hieß konkret: zu seiner `Theorie der tonalen Funktionen` und seiner sdualistischen Begründung der Harmonie- 
lehre . Johannes Schreyer und seine Harmonielehre können dabei als symptomatisch für die erste Generation der Riemann-Rezeption betrachtet werden: Schreyer lehnt die dualistische Bezeichnung des Molldreiklangs ab, übernimmt andere `dualistische` Rudimente jedoch unbesehen in seine vermeintlich ımonistische` Theorie. ${ }^{1}$ Von weitaus größerem Interesse als der `Dualismus` war für Schreyer die Riemannsche `Theorie der tonalen Funktionen . Diese Theorie war nur vordergründig eine neue Art der analytischen

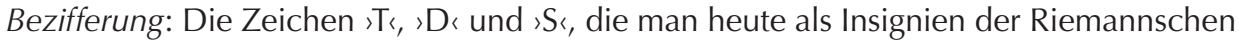
Theorie zu betrachten gewohnt ist, sind eine vergleichsweise späte Erscheinung in der Riemannschen Harmonielehre: ${ }^{2}$ Im Kern war Riemanns Reform der Harmonielehre ein - in Absetzung von der »Generalbaßharmonielehre « ${ }^{3}$ entstandenes - neues Verständnis zentraler musiktheoretischer Kategorien wie Akkord, Tonalität und Modulation. An der Harmonielehre Schreyers läßt sich jedoch wie an keiner zweiten ablesen, was für die Riemann-Anhänger die eigentliche Attraktion der Riemannschen Theorie ausmachte: ihr analytisches Potential. Die Riemannsche Bezifferung, die sich in einem radikal reduktionistischen Sinne zur übersichtlichen Skizzierung komplexer harmonischer Abläufe anwenden ließ ${ }^{4}$, sah Schreyer den alten Bezifferungssystemen in ihrer Bedeutung für die praktische Analyse weit überlegen: Die klassischen Fundamentalbaß- und Stufentheorien konnten mit ihrem «klassischen Modulationsbegriff des 18. und 19. Jahrhunderts die spätromantische Harmonik Wagners und Liszts nur als Unmenge von Modulationen begreifen und dementsprechend kompliziert beziffern; die Riemannsche Funktionstheorie dagegen mit ihrem neuen, mit Zwischendominanten operierenden Modulationsbegriff, erlaubte es, längere Abschnitte zu analysieren, ohne beständig von Modulationen sprechen zu müssen (vgl. Holtmeier 2004).

Im Vorwort zu seiner Harmonielehre, die den Versuch darstellt, die Riemannsche Theorie zu einem praktischen Lehrgang umzuformen, schreibt Schreyer in diesem Sinne: »Es kam dem Verfasser besonders darauf an, dem Leser so bald als möglich die Formel

$$
\mathrm{T} \mathrm{S}^{6} \mathrm{D}^{7} \mathrm{~T}
$$

zu erklären und den Nachweis zu führen:

1. daß alle in der Musik gebrauchten Zusammenklänge nur Absenker dieser Stammklänge sind und

2. daß es möglich ist, mit dieser schlichten Formel die kompliziertesten modernen Kompositionen zu analysieren«(Schreyer 1905, IV).

1 Zum Beispiel die prinzipiell dualistischen Riemannschen »Parallelklänge«. Zur Entstehung und Entwicklung des »Dualismus« vgl. Klumpenhouwer 2002; Holtmeier 2004.

2 Sie erscheinen erstmals in seiner Schrift Vereinfachte Harmonielehre oder die Lehre von den tonalen Funktionen der Harmonie (London 1893), also zwanzig Jahre nach Riemanns ersten Veröffentlichungen zur Harmonielehre. Ludwig Holtmeier hat gezeigt, daß man in der Einführung der Funktionszeichen sogar eine Wende im Riemannschen Denken sehen kann (vgl. Holtmeier 2004).

3 Mit `Generalbaßharmonielehre` bezeichneten Riemann und seine Anhänger die Lehrbücher in stufentheoretischer und Fundamentalbaß-Tradition.

4 Am deutlichsten hat dies damals der Riemann-Anhänger Eugen Schmitz in seiner Harmonielehre (1911) geschildert. Vgl. Diergarten 2004. 

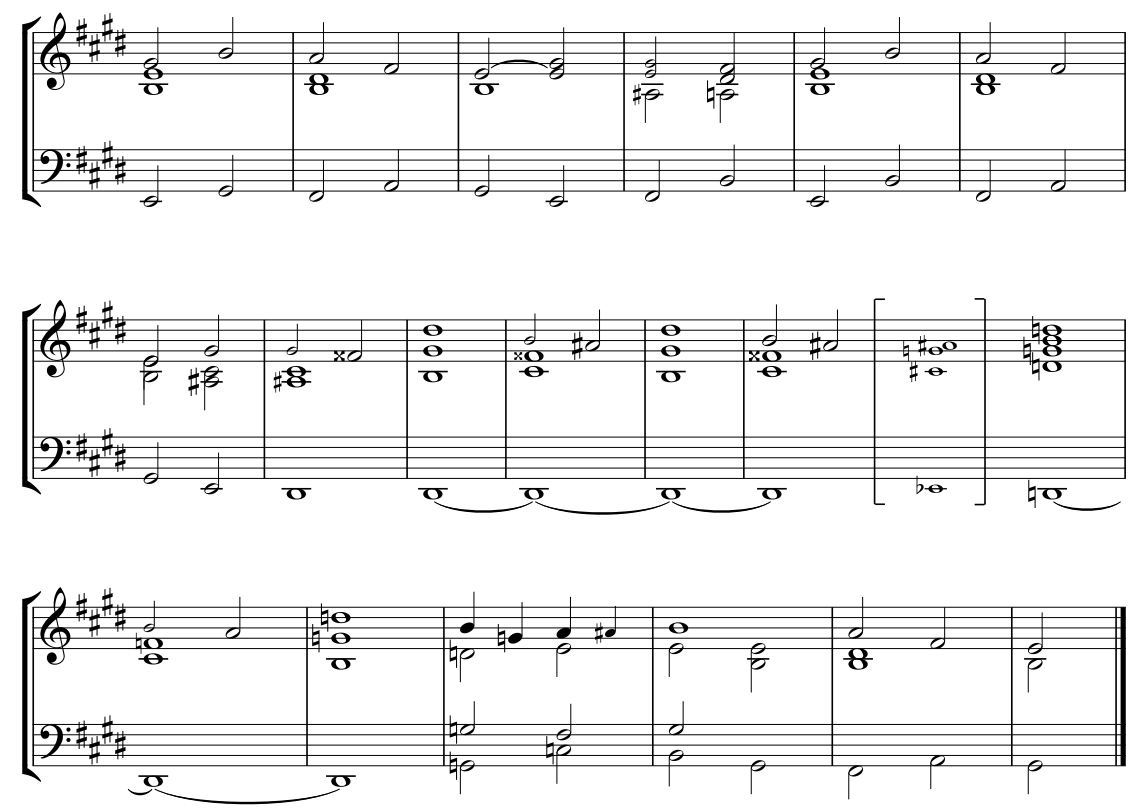

Beispiel 1

Unter `Analyse « versteht Schreyer die Anfertigung reduktionistischer Harmonieskizzen, in denen der »harmonische Extrakt polyphoner Kompositionen« in Form eigenständiger Nachbildungen dargestellt wird. Um eine derartige Reduktion der ersten "Consolation« von Franz Liszt handelt es sich bei Notenbeispiel 1 (ebd., S. 210).

Schreyer reduziert den Klaviersatz auf ein akkordisches Gerüst und beziffert dieses mit den Riemannschen Funktionszeichen, deren korrekter Gebrauch ihm teilweise noch Schwierigkeiten bereitet. ${ }^{5}$ Besondere Aufmerksamkeit verdienen jedoch die Takte 8ff. des Beispiels 1, die Schreyer mit einem einzigen `D، im Baß beziffert, gefolgt von drei Punkten, die die 'Verlängerung`, die `Prolongation` dieser Funktion bezeichnen: Das Verständnis von `Funktion`, das hinter dieser Bezifferung steht - die Projektion von Kadenzmodellen auf verschiedene harmonische Ebenen - ist nicht weit entfernt vom Begriff der `Stufe`, den Heinrich Schenker im Jahre 1906 prägen sollte. ${ }^{6}$ In diesem Sinne schrieb ein Kritiker über Schreyers Analysen:

Solches schöpferisches Analysieren erzieht in außerordentlicher Weise zum Hören von Zusammenhängen und zur Auffassung des Gesetzmäßigen. In der Benützung dieser Analyse berührt sich Schreyer sehr stark mit Heinrich Schenker in Wien, der in seinen

5 Vgl. etwa die Ausweichung nach gis-Moll in der zweiten Zeile, die in der Bezifferung nicht korrekt wiedergegeben wird.

6 »ohannes Schreyers Reduktionen zeigen, daß die frühe Funktionstheorie durchaus so etwas wie eine sSchichtenlehre kannte« (Holtmeier 2003. Vgl. auch Christensen 1982). 
theoretischen Schriften den Begriff der »Urlinie« einführt und in seinen Heften »Der Tonwille« Analysen von Meisterkompositionen mit ihren Urlinienbildern mitteilt. Das Urlinienbild verhält sich zur ausgeführten Komposition wie das Thema zur Variation, es stellt ganz ähnlich wie die Schreyersche Analyse nur den gesetzmäßigen Verlauf der Grundbewegungen dar (Stier 1929).

In sämtlichen Auflagen seiner Schrift bezeichnete Johannes Schreyer die Analyse als "Ziel und den Mittelpunkt» seiner Harmonielehre. Bemerkenswert ist dabei die Tatsache, daß die Analyse bei Schreyer nicht dazu dient, einzelne musiktheoretische Sachverhalte zu illustrieren, wie dies im 19. Jahrhundert üblich wurde: Die Beispielanalysen dienen tatsächlich der Anleitung zur Analyse. Diese neue, außergewöhnliche Rolle der Analyse erklärt sich aus dem Selbstverständnis der Schreyerschen Harmonielehre, die Schreyer als »Anleitung zum Verständnis und Genuß musikalischer Kunstwerke« sowie als Grundlage »einer rationellen Lehre des Vortrags« bezeichnete.

\section{Reformpädagogik}

Um die Wende zum 20. Jahrhundert erwachte in Deutschland und in weiten Teilen Europas eine Fülle kulturkritischer Bewegungen. Für einzelne Strömungen dieser großen und weit verzweigten Bewegung haben sich Begriffe wie `Jugendbewegung`, 'LebensreformBewegung`, >Reformpädagogikı oder ‘Kunsterziehungsbewegung` etabliert. ${ }^{`}$ Von besonderer Bedeutung in diesem Zusammenhang ist die sogenannte sKunsterziehungsbewegung`, der kunst-pädagogische Zweig der Reformpädagogik: ${ }^{8}$ Wesentliches Anliegen der Kunsterzieher war die Reform des künstlerischen und gymnastischen Unterrichts, mit der man der drohenden Selbstentfremdung des Menschen begegnen wollte. Diesem Reformvorhaben zentral war die Forderung nach sganzheitlicher und sschöpferischer Pädagogik, nach verstärktem Eingehen auf das Kind mit seinen spezifischen Bedürfnissen, weiterhin die Hinwendung zu Laien- und Volkskunst sowie die Betonung von Begriffen wie `Ausdruckı und `Körperı. Anhand von fünf Zitaten aus der Schreyerschen Harmonielehre sollen im folgenden schlaglichtartig die Spuren beleuchtet werden, die die Reformpädagogik im Werk Schreyers und in einem großen Teil der deutschen Musiktheorie der ersten Jahrhunderthälfte hinterlassen hat.

Erstes Zitat: »Eine Anleitung zum Genuß musikalischer Kunstwerke«. Schreyer verurteilt in seiner Harmonielehre - ganz im reformpädagogischen Jargon - musiktheoretische Lehrbücher, die »über abstrakte Begriffe und fantasielähmende Nörgeleien « ${ }^{9}$ nicht hinauskommen und die bevorzugen, »fortwährend zu reflektieren, anstatt spontan Gefühltes in der Sprache unserer Zeit, wenn auch unbeholfen und stammelnd auszu-

7 Einführende Darstellungen in diese Thematik finden sich in Hodek 1977 und Höckner 1927.

8 Geprägt wurde der Begriff bei den in den Jahren 1903 bis 1905 in Dresden, Weimar und Hamburg abgehaltenen »Kunsterziehungstagen«, die sich der Reihe nach den Themen »Bildende Kunst» (Dresden), »Dichtkunst« (Weimar) und »Musik und Gymnastik« (Hamburg) widmeten. Vgl. Hein 1991.

9 Schreyer, 1911, 107. 
drücken. ${ }^{10}$ Schreyer kritisiert weiterhin die Lehrbücher, die »nicht von den Erfahrungen des Hörens, sondern von Systemen und starren Regeln « ${ }^{11}$ ausgehen. In seinem eigenen Lehrbuch bietet Schreyer bereits 1903 eine moderne Lösung für dieses Problem an: eine Handwerkslehre, deren Regeln aus der Analyse erst gewonnen werden, sowie einen Analysekurs, der versucht, das theoretisch vermittelbare Allgemeine mit dem HistorischIndividuellen zu verbinden.

Zweites Zitat: »Die zerstückelte Art, die Kunst zu behandeln«. Mit dieser abfälligen Charakterisierung meint Schreyer »die Gegenüberstellung von Harmonielehre und Kontrapunkt, von strengem und freiem Satze. «12 Er behauptet, es ließe sich "geschichtlich nachweisen, daß diese Anschauungen die Hauptschuld tragen an der 150 Jahre währenden Stagnation der Methode und dem verwirrenden Zwiespalt zwischen Theorie und Praxis « ${ }^{13}$ Gleichzeitig mit dem Vorwurf der falschen Trennung der Disziplinen richtet sich Schreyers Kritik jedoch auch gegen deren falsche Vermischung, etwa gegen die Ersetzung einer swirklichen ‘ Harmonielehre durch einen Katalog von kontrapunktischen Regeln. Schreyer entwirft so in seiner Harmonielehre ein äußerst differenziertes Bild von sintegrativer musiktheoretischer Unterweisung, in der die einzelnen Fächer zwar institutionell getrennt bleiben, jedoch füreinander durchlässig und aufeinander abgestimmt sind.

Drittes Zitat: das Gehör als »Maßstab für die Beurteilung eines Kunstwerks«. Ein zentrales Anliegen der Schreyerschen Harmonielehre ist die Schulung des Gehörs, die für Schreyer in erster Linie eine Schulung des (so würde man heute sagen:) musikalischen `Hör-Verstehens` darstellt. Für die eher stechnische`Seite der Gehörbildung (vielleicht sollte man, um nicht selbst in den kulturkritischen Jargon zu verfallen, besser sagen: für die eher ssportive` Seite der Gehörbildung) verweist Schreyer auf systematische Übungen in damals international gängigen Lehrbüchern ${ }^{14}$. Das Gegenstück der Erziehung zur musikalischen Genußfähigkeit war für Schreyer die Erziehung zu musikalischer Ausdrucksfähigkeit. Alle von der Lebensphilosophie inspirierten Reformbewegungen sahen in ausdrucksvoller Tätigkeit ein zentrales Element menschlichen Daseins. Auch den Kunsterziehern ging es weniger um die Ausbildung sogenannter stechnischer Fertigkeiten als um die Förderung eigener, ausdrucksvoller Produktion, wenn diese auch dilettantisch und unbeholfen sein sollte.

Viertes Zitat: „Die Kräfte des Elementarschülers«. Mit dem steten Wachstum der bürgerlichen Kultur im 19. Jahrhundert kam das Musizieren von Laien und Amateuren zu ungeahntem Aufschwung. Die Laien waren die Zielgruppe der vielen »Allgemeinen Musiklehren « und Harmonielehren dieser Zeit. ${ }^{15}$ Daß auch Johannes Schreyer beim Verfassen seiner Harmonielehre einen solchen Personenkreis im Auge hatte, zeigt sich nicht zuletzt an der Auswahl musikalischer Beispiele, die zum größten Teil einem ganz be-

10 Schreyer, 1911, $106 f$.

11 Schreyer, 1903, 1.

12 Schreyer 1903, 11.

13 Ebd.

14 Schreyer nennt die Bücher von Tufts/Holt, Ritter, Lavignac, Johne, Riemann und Roger-Ducasse.

15 Vgl. Wason 2002, 63. 
stimmten Feld der Klavierliteratur entnommen sind: Es sind die ersten Stücke, die sich ein junger Klavierschüler erarbeitet - Sonatinen von Clementi, Etüden von Czerny, Lieder ohne Worte, kleinere Klavierstücke von Chopin. Schreyer betont den didaktischen Wert dieser Literatur und schreibt etwa über die Etüden Czernys:

Obgleich der musikalische Wert dieser lediglich für technische Zwecke komponierten
"Übungen « nur ein geringer ist, so läßt sich doch vieles aus ihnen lernen, weil sie im
Rahmen der achttaktigen Periode die Dur-Kadenz von allen Seiten umkreisen und die
drei Funktionen der Harmonie sowohl in Akkordform als auch figuriert zeigen. Sie eig-
nen sich nicht nur sehr gut zur Vorbereitung auf die Analyse wirklicher Meisterwerke,
sondern lassen sich für fantasiebegabte Schüler auch noch in anderer Weise nutzbar
machen. ${ }^{16}$

Schreyer betont darüber hinaus, daß die Fähigkeit, die analysierten Werke auf dem Klavier darzustellen, eine wichtige Ergänzung zur Analyse bedeutet.

Fünftes Zitat: »Abstrakte, rein verstandesmäßige Erklärungen sind möglichst vermieden worden ${ }^{17}$. An einigen Stellen der Harmonielehre Schreyers tritt deutlich eine rationalitätskritische Haltung zutage, wie sie charakteristisch für beinahe alle kulturkritischen Bewegungen ist. Die Kritik an »rein verstandesmäßige[n] Erklärungen« und am `Theoretisieren ist jedoch bei Schreyer in erster Linie eine Kritik an den Harmonielehren der Generalbaß-Tradition: Harmonielehren, die Stimmführungsreglements lehren, dabei auf musikalische Beispiele verzichten und dadurch - so könnte man sagen - die musikalische Wahrnehmung außer acht lassen. So betrachtet, hat die anti-rationalistische Argumentation durchaus ihr historisches und fachliches Recht. Die Quintessenz der Schreyerschen und jeder anderen lebensphilosophisch inspirierten Methodik könnte man als `Erziehung zur Erfahrung، bezeichnen, in der man auch noch heute, trotz aller Vorbehalte, die sich rückblickend der Lebensphilosophie gegenüber ergeben mögen, deren große Errungenschaft sehen mag. Es ist jedoch die enge Bindung an das anti-rationalistische Umfeld der lebensphilosophischen Reformpädagogik, die die deutsche Musiktheorie anfällig machte für die svölkische` und letztendlich theoriefeindliche Entwicklung in den 30er und 40er Jahren (vgl. Holtmeier 2003).

Schreyers Harmonielehre rührte zum Zeitpunkt ihres Erscheinens an Grundfragen des musiktheoretischen und kulturtheoretischen Diskurses, die hier unter den Begriffen $>$ Riemann-Rezeption` und `Reformpädagogikı zusammengefaßt sind. Das Zusammentreffen dieser beiden Faktoren war kein Zufall: Wollte man das Lebenswerk Riemanns unter einen großen Bogen fassen, so könnte man sagen, daß es eine Wende gegen die alte physikalische Theorie und gegen die neuere physiologische Theorie (Helmholtzscher Prägung) hin zu einer psychologischen Theorie vollzog: Eine neukantianische Wende snach innen . Nicht umsonst fand Riemanns Lebenswerk den Abschluß in dem ehrgeizigen Projekt einer `Lehre von den Tonvorstellungenı. Es scheint, als löse erst die Be-

16 Schreyer 1911, 115.

17 Harmonielehre 1905, IV. 
hauptung Ernst Kurths: »Funktion ist energetisch «18, ein Potential der Funktionstheorie ganz ein, das bei Riemann selbst noch nicht konsequent zu Ende geführt ist. Riemanns Theorie ebnete jedoch erst den Weg für die reine sInnerlichkeit` und Psychologie späterer deutscher Theorien. ${ }^{19}$ Vor diesem Hintergrund ließe sich die Behauptung aufstellen, daß die deutsche Ideologie der sInnerlichkeit die unterschwellige Verbindung zwischen Riemann-Rezeption und Reformpädagogik darstellt.

\section{Literatur}

Christensen, Thomas: »The Schichtenlehre of Hugo Riemann«, In Theory Only 6 (1982), 37-47.

Diergarten, Felix: »Eugen Schmitz«, in: MGG (Personenteil) (2004).

Hein, Peter Ulrich: Transformation der Kunst. Ziele und Wirkungen der deutschen Kulturund Kunsterziehungsbewegung, Köln 1991.

Höckner, Hilmar: Die Musik in der deutschen Jugendbewegung, Wolfenbüttel 1927.

Hodek, Johannes: Musikalisch-pädagogische Bewegung zwischen Demokratie und Faschismus. Zur Konkretisierung der Faschismus-Kritik Th. W. Adornos, Weinheim 1977.

Holtmeier, Ludwig: „Von der Musiktheorie zum Tonsatz. Zur Geschichte eines geschichtslosen Faches", Zeitschrift der Deutschen Gesellschaft für Musiktheorie 1 (2003, 11-34 Printausgabe in diesem Band).

Ders.: »Zur Riemann-Rezeption«, in: Helga de la Motte-Haber (Hg.), Handbuch der Systematischen Musikwissenschaft, Bd. 2: Musiktheorie, hg. von Helga de la Motte-Haber u. Oliver Schwab-Felisch, Laaber 2004.

Klumpenhouwer, Henry: »Dualist tonal space and transformation in nineteenth-century musical thought", in: Thomas Christensen (Hg.), The Cambridge History of Western Music Theory, Cambridge 2002, 456-476.

Kurth, Ernst: Die Romantische Harmonik und ihre Krise in Wagners Tristan, Berlin ${ }^{3} 1923$.

Riemann, Hugo: Vereinfachte Harmonielehre oder die Lehre von den tonalen Funktionen der Harmonie, London 1893.

Schmitz, Eugen: Harmonielehre als Theorie, Ästhetik und Geschichte der musikalischen Harmonie, Kempten 1911.

Schreyer, Johannes: Lehrbuch der Harmonie und der Elementarkomposition, Dresden 1905, Leipzig ${ }^{2} 1911$.

Schreyer, Johannes: Von Bach bis Wagner. Beiträge zur Psychologie des Musikhörens, Dresden 1903.

18 Vgl. Ernst Kurth, Die Romantische Harmonik und ihre Krise in Wagners Tristan, Berlin ${ }^{3} 1923,52$. Vgl. auch 11 und 45.

19 Vgl. Diergarten, »Schmitz« (Anm. 5). 
Stier, Alfred: »Johannes Schreyer zum Gedächtnis«, Zeitschrift für Kirchenmusiker, Nr. 10 (1929), 175-77.

Wason, Robert W.: »Musica Practica. Music Theory as Pedagogy«, in: Thomas Christensen (Hg.), The Cambridge History of Western Music Theory, Cambridge 2002, 46-77. 